\title{
Determination of Malaria Parasite Concentration using Image Processing
}

\author{
Namitha Prasad \\ Dept. of ECE, \\ National Engineering College, \\ Kovilpatti, India \\ F. Swanline \\ Dept. of ECE, \\ National Engineering College, \\ Kovilpatti, India
}

\author{
N. Nithyashree \\ Dept. of ECE, \\ National Engineering College, \\ Kovilpatti, India \\ M. Sathish Kumar M.E \\ Dept. of ECE, \\ National Engineering College, \\ Kovilpatti, India
}

\begin{abstract}
Malaria may be a severe communicable disease caused by feminine genus Anopheles mosquito. In 2016 alone 216 million cases of protozoal infection occurred worldwide and 445000 died. Thus treatment ought to be initiated as shortly as attainable. Except species of infecting parasite, concentration of protozoal infection decides the course of correct treatment. Patients with severe protozoal infection want intensive parental care till the parasite density falls below $1 \%$ and that they will tolerate oral medical care. If blood disorder exceeds $10 \%$ then transfusion is also necessary. A general structure of image process is employed to search out the concentration of malaria parasite. The skinny blood smear pictures of Giesma stained square measure processed. The system uses intensity as a feature to search out the malaria parasite. The image is pre-processed and binarized and metameric to search out the circles of interest wherever infected erythrocytes square measure found. This investigates the employment and application of Digital Image process for detecting malaria parasite and deciding its concentration exploitation microscopic color pictures.
\end{abstract}

Keywords - Protozoal infection, blood smear, Giesma stained, Digital Image process.

\section{INTRODUCTION}

Malaria is one amongst fatal diseases worldwide and might cause death chop-chop if it's untreated. In 2015, 214 million cases of protozoal infection were calculable, that caused around 438,000 deaths. Protozoal infection is rife within the tropical and semitropical regions close to the equator like Asia, Africa, and geographical area. The sickness is caused by genus Plasmodium parasites and transmitted by the feminine anopheles' dipterous insect.

There square measure primarily four species of genus Plasmodium that infect humans are: Plasmodium vivax, Plasmodium Falciparum, Plasmodium Ovale and Plasmodium protozoal infection. Among these species, Plasmodium vivax causes vital health problems worldwide. in line with world health organization, there have been total 13.8 million cases were calculable globally in 2015 . Since 2010, UN agency has recommended protozoal infection check diagnostic assay by either light-weight research or fast diagnostic test [1]. RDT is employed wherever research or well-trained microscopists is not available; this methodology provides instant results however main disadvantage of RDT is that parasite density cannot be calculable. RDT may be a medical assay that's fast and straight forward to perform. RDTs square measure appropriate for preliminary or emergency medical screening and to be used in medical facilities with restricted resources. Digital image process is that the use of pc algorithms to perform image process on digital pictures. As a subcategory or field of digital signal process, digital image process has several benefits over analog image process. It permits a way wider vary of algorithms to be applied to the input file and might avoid issues like the build-up of noise and signal distortion throughout process. Since pictures square measure outlined .Over 2 dimensions (perhaps more) digital image process is also modeled within the type of four-dimensional systems.

\section{II.PROPOSED WORK}

\section{A. IMAGE ACQUISITION}

The blood smear images were acquired from the Centre for Disease Control (CDC) website and captured from the Reference Laboratory of Malaria, in Sudan Ministry of Health.

\section{B. IMAGE PRE-PROCESSING}

The goal of this step is to form the acquired images more suitable for subsequent processes, mainly image segmentation and have extraction. Basically, there are three main objectives for image pre-processing.

- One is to resize the image for the needs of either magnifying the image through digital zooming, or reducing the image size so as to hurry up processing.

- The second objective of image pre-processing is to scale back or eliminate noise from the acquired image.

- The third objective is to reinforce the image contrast for visual evaluation. All images are rescaled to possess an equivalent size using the inbuilt MATLABTM function in resize. 
Since all CDC images have a resolution of $300 \times 300$ pixels, therefore they are doing not got to be rescaled. the dimensions of CDC images are adopted to be the quality image size of the pre-processed images

Images are converted from RGB to gray scale to scale back the time interval. RGB to gray conversion is completed by using the inbuilt MATLABTM function rgb2gray, which converts RGB, images to grayscale by eliminating the hue and saturation information while retaining the luminance.

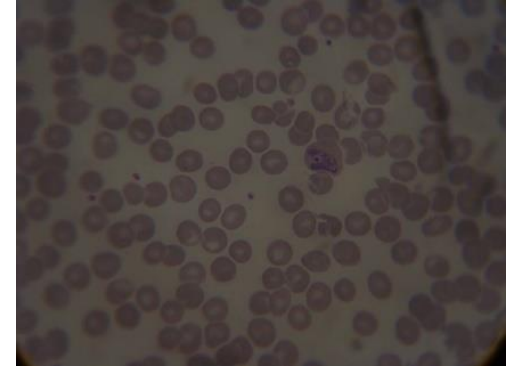

Fig.1 RGB image

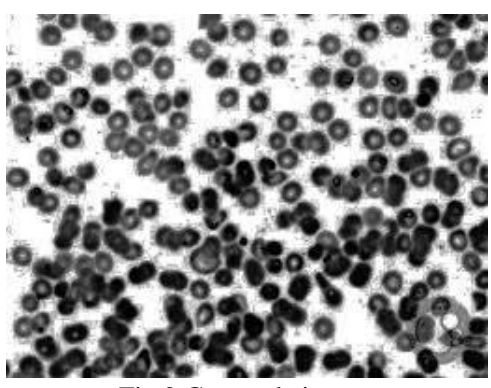

Fig.2 Grayscale image

\section{THRESHOLDING}

Thresholding is that the simplest method of image segmentation. From a grayscale image, thresholding are often wont to create binary images.

The simplest method of image segmentation is named the thresholding method. This method is predicated on a cliplevel (or a threshold value) to show a gray-scale image into a binary image.

The key of this method is to pick the edge value (or values when multiple-levels are selected). Several methods are

- Maximum entropy method,

- Balanced histogram thresholding,

- Otsu's method (maximum variance) and

- K-means clustering.

Recently, methods are developed for thresholding computerized tomography (CT) images. The key idea is that, unlike Otsu's method, the thresholds are derived from the radiographs rather than the (reconstructed) image.

New methods suggested the usage of multi-dimensional fuzzy rule-based non-linear thresholds. In these works, decision over each pixel's membership to a segment is predicated on multi-dimensional rules derived from symbolic logic and evolutionary algorithms supported image lighting environment and application.

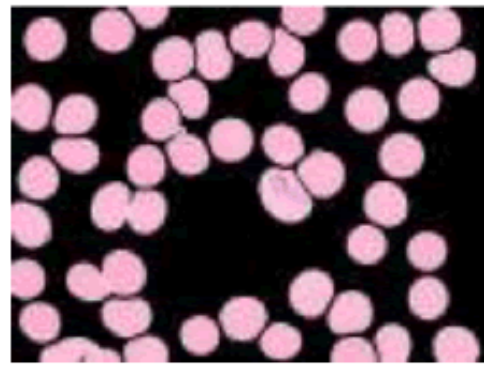

Fig.3 Malaria parasite

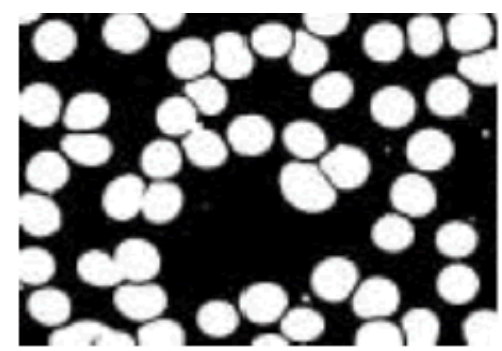

Fig.4 Thresholded image

\section{D.OTSU'S THRESHOLDING}

In laptop vision and image process, Otsu's technique, named once Nobuyuki Otsu (Otsu Nobuyuki), is utilized to perform automatic image thresholding. at intervals the best kind, the formula returns one intensity threshold that separate pixels into 2 categories, foreground and background. This threshold is set by minimizing intra-class intensity variance, or equivalently, by increasing inter-class variance. Otsu's technique is also a one-dimensional separate analog of Fisher's Discriminant Analysis, is alleged to Jenks optimization technique, and is sort of a globally best kmeans performed on the intensity bar chart.

Algorithm

1. Figure bar chart and possibilities of each intensity.

2. Noted initial ai(0) and bi(0).

3. Step through all doable thresholds $\mathrm{t}=0$ most intensity

1. Update ai and bi.

2. Figure threshold.

\section{E. BINARIZATION}

Image binarization is that the method of taking a grayscale image and changing it to black-and-white, primarily reducing the data contained at intervals the image from 256 reminder grey to 2 : black and white, a binary image. this is often typically cited as image thresholding, though thresholding could turn out pictures with quite two levels of grey. it is a kind or segmentation, whereby an image is split into constituent objects. This is often a task ordinarily performed once associate attempt to extract an object from an image. However, like several image process operations, it isn't trivial, and is entirely captivated to the content at intervals the image. 


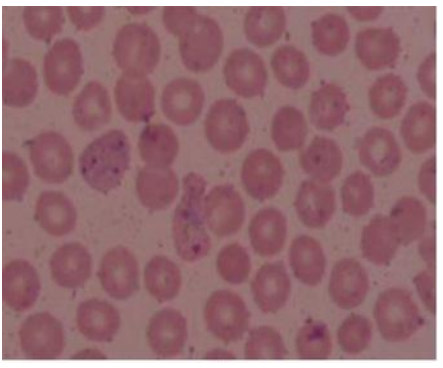

Fig.5 Malaria parasite

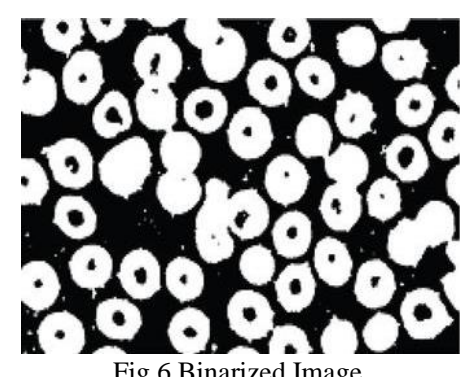

Fig.6 Binarized Image

\section{F. MORPHOLOGY}

Morphological image process is also a group of non-linear operations related to the shape or morphology of options in an exceedingly image. Morphological operations bank solely on the relative ordering of pixel values, not on their numerical values, and therefore square measure particularly suited to the process of binary pictures. Morphological operations can also be applied to greyscale pictures such their lightweight transfer functions square measure unknown and therefore their absolute pixel values square measure of no or minor interest.

Morphological techniques probe an image with a little form or templet referred to as a structuring part. The structuring part is positioned at intervals the smallest amount doable locations within the image and it's compared with the corresponding neighborhood of pixels. Some operations take a look at whether or not the part "fits" at intervals the neighborhood, whereas others take a look at whether or not it "hits" or intersects the neighborhood.

A morphological operation on a binary image creates a replacement binary image throughout that the pixel options a non-zero worth as long because the take a look at is winning at that location at intervals the input image.

The structuring part is also a tiny low binary image, i.e. a little matrix of pixels, every with a value of zero or one:

- The matrix dimensions specify the scale of the structuring part.

- The pattern of ones and zeros specifies the shape of the structuring part.

- Associate in origin of the structuring part is often one in every of its pixels, though typically the origin square measure typically outside the structuring part.

More formal descriptions and samples of however basic morphological operations work square measure given at intervals the multimedia Image process Reference (HIPR) developed by Dr. R. Fisher et al. at the Department of AI at intervals the University of Edinburgh, Scotland, UK.

The erosion of a binary image $f$ by a structuring element $s$ (denoted $\mathrm{f} s$ ) produces a new binary image $\mathrm{g}=\mathrm{f} \quad \mathrm{s}$ with ones altogether locations $(\mathrm{x}, \mathrm{y})$ of a structuring part's origin at that that structuring element $s$ fits the input image $f$, i.e. $g$ $(\mathrm{x}, \mathrm{y})=1$ is $\mathrm{s}$ fits $\mathrm{f}$ and zero otherwise, continuation for all pixel coordinates $(\mathrm{x}, \mathrm{y})$.

Erosion removes small-scale details from a binary image but simultaneously reduces the size of regions of interest, too. By subtracting the eroded image from the original image, boundaries of each region can be found: $b=f-(f \ominus s$ ) where $f$ is an image of the regions, $s$ is a $3 \times 3$ structuring element, and $b$ is an image of the region boundaries.

Morphological filtering of a binary image is conducted by considering compound operations like gap and shutting as filters. they will act as filters of form. for instance, gap with a disc structuring component smooths corners from the within, and shutting with a disc smooths corners from the skin. however additionally these operations will filter from a picture any details that area unit smaller in size than the structuring component, e.g. gap is filtering the binary image at a scale outlined by the dimensions of the structuring component. solely those parts of the image that work the structuring component area unit glided by the filter; smaller structures area unit blocked and excluded from the output image. The dimensions of the structuring component are most significant to eliminate clanging details however to not harm objects of interest.

There are several commands employed in morphological method. Some commands are

- Disk

- Spur

- Perimeter

- Skeleton

The pruning algorithm may be a technique employed in digital image process supported mathematical morphology. It's used as a complement to the skeleton and cutting algorithms to get rid of unwanted parasitic elements (spurs). The bwperim operate determines the perimeter pixels of the objects in a very binary image. A constituent is taken into account a fringe constituent if it satisfies each of those criteria

- The constituent is on.

- One (or more) of the pixels in its neighborhood is off. In digital image process, morphological skeleton may be a skeleton (or medial axis) illustration of a form or binary image, computed by means that of morphological operations.

\section{G. WATERSHED SEGMENTATION}

It was introduced by Luc Vincent and Pierre Soille is predicated on the idea of "immersion". Each and every local minima of a gray-scale image I which may be regarded as surface which contain a hole and therefore the surface is immersed out into water. Then, ranging from the minima of lowest intensity price, the water can more and more refill completely different construction basins of image (surface). Conceptually, the rule then builds a dam to avoid a scenario that the water returning from 2 or a lot of completely different local minima would be incorporate. At the tip of this immersion method, every local minimum is completely confined by dams such as watersheds of image (surface). 
The watershed remodel has been wide employed in several fields of image process, together with medical image segmentation, because of the quantity of benefits that it possesses: it's a straightforward intuitive methodology, it's quick and might be parallelized associate degreed an nearly linear speeding was rumored for variety of processors up to 64) and it produces a whole division of Image Segmentation using Watershed remodel Amandeep Kaur, Aayushi Image Segmentation using Watershed remodel 6 printed By: Blue Eyes Intelligence Engineering \& Sciences Publication Pvt. Ltd. the image in separated regions even though the distinction is poor, so avoiding the requirement for any quite contour connexion. It's acceptable to use this methodology to section the high-resolution remote sensing image.

\section{G. CONCENTRATION}

Malaria parasite image is taken as area. The amount of area depends on the depth of the infected cells. Concentration is measured by the amount of infected cell and total area of the image. Concentration of malaria parasite is done on Matlab.

Table-1: Concentration range and types of plasmodium

\begin{tabular}{|c|c|}
\hline CONCENTRATION & TYPES \\
\hline Concentration $<3$ & $\begin{array}{c}\text { Plasmodium } \\
\text { Falciparum }\end{array}$ \\
\hline $5<$ Concentration $<10$ & Plasmodium Vivax \\
\hline $10<$ Concentration $<20$ & Plasmodium Knowlesi \\
\hline $20<$ Concentration $<30$ & Plasmodium ovale \\
\hline Concentration $>30$ & Plasmodium Malaria \\
\hline
\end{tabular}

\section{RESULT}

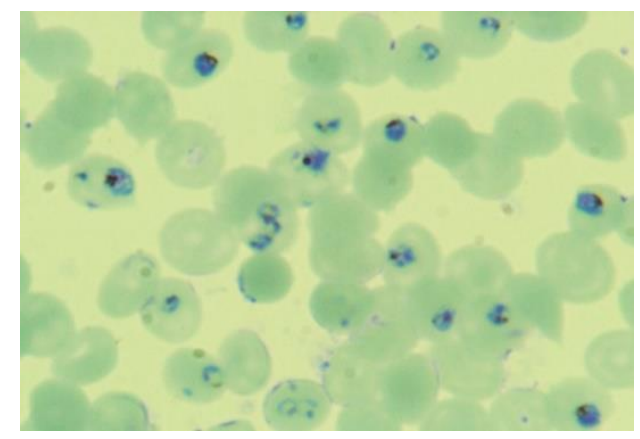

Fig.7 Microscopic image of malaria parasite

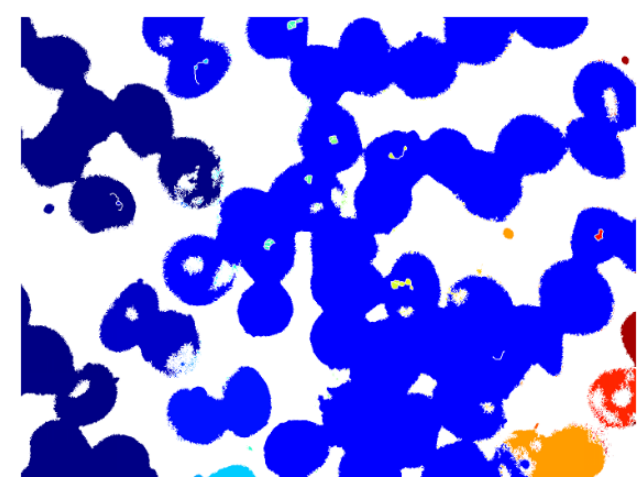

Fig.8 Segmented image using matlab

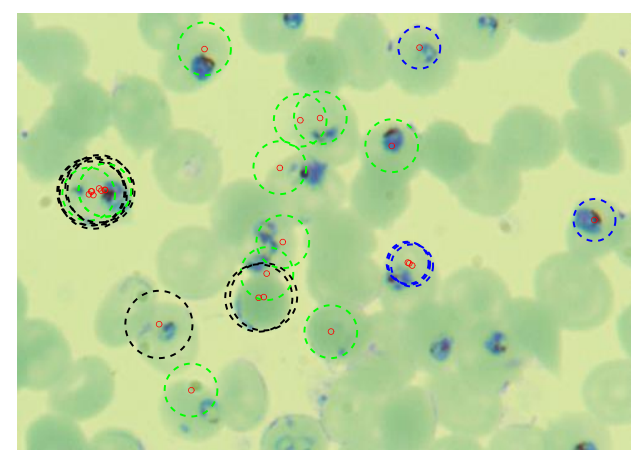

Fig.9 Concentrated image

\section{CONCLUSION}

Pathologists manually find Plasmodium vivax victimization microscopes which may cause human error. Also, these results are hard to breed whenever necessary. Automatic identification of Plasmodium vivax eliminates the human error and additionally reduces the time process. And additionally such results may be reproduced simply whenever required. Based on the types of plasmodium, the concentration of malaria parasite is determined in this proposed work.

\section{REFERENCE}

[1] WHO. World malaria report 2017. World Health Organization, Geneva; 2017. Tech Reports.

[2] C. Di Ruberto, A. Dempster, S. Khan, and B. Jarra, "Automic thresholding of infected blood images using granlometry and regional extrema", 441-444, 2000 IEEE

[3] Halim S, BretschneiderT.R, YikunLi, Preiser P.R.," Estimating malaria parasitaemia from blood smear images", proceedings of the 9 th international conference on control, automation, robotics and vision, PP.16, Dec.2006.

[4] Varsha Waghmare, Syed Akhter," Image analysis based system for automatic detection of malarial parasite in blood images", International Journal of Science \& Research(IJSR), ISSN(Online):23197064, July, 2015.

[5] P. Pratim Acharjya and M. Santiniketan," Watershed Segmentation based on Distance Transform and Edge Detection Techniques", International Journal of Computer Applications (0975 - 8887) Volume 52- No.13, August 2012 